\title{
ONE-RELATOR QUOTIENTS AND FREE PRODUCTS OF CYCLICS
}

\author{
BENJAMIN FINE, JAMES HOWIE AND GERHARD ROSENBERGER
}

(Communicated by Bhama Srinivasan)

\begin{abstract}
It is proven that the Freiheitssatz holds for all one-relator products of cyclic groups if the relator is cyclically reduced and a proper power. The method of proof involves representing such groups in $\mathrm{PSL}_{2}(\mathbf{C})$ and is a refinement of a technique of Baumslag, Morgan and Shalen. The technique allows the extension of the Freiheitssatz result to many additional one-relator products.
\end{abstract}

1. Introduction. A one-relator product of a family of groups $\left(A_{i}\right), i \in I$, is the quotient $\left(* A_{i}\right) / N(R)$ where $R$ is a cyclically reduced word and $N(R)$ is its normal closure in the free product $*_{i} A_{i}$. $R$ is called the relator. A one-relator group is just a one-relator product of free groups. In general one-relator products share many properties with one-relator groups [3].

In the present paper we give a version of the Freiheitssatz for a certain class of one-relator products of cyclic groups. In particular we show that if each $A_{i}$ is cyclic and the relator is a proper power then the subgroup generated by any proper subset of the generators is the obvious free product of cyclics. This answers a conjecture of J. Howie [3]. The method of proof involves representing such groups in $\mathrm{PSL}_{2}(\mathbf{C})$ and is a refinement of a technique of Baumslag, Morgan and Shalen [1]. This technique was used in [1] to investigate the class of generalized triangle groups which in the present context can be described as one-relator products of two cyclic groups. The technique actually yields a stronger result-a Freiheitssatz for one-relator products of groups which admit faithful representation in $\mathrm{PSL}_{2}(\mathbf{C})$.

2. Freiheitssatz. Our main result is the following.

THEOREM 1. Suppose the group $G$ is given by

$$
G=\left\langle a_{1}, a_{2}, \ldots, a_{n} ; a_{1}^{e_{1}}=a_{2}^{e_{2}}=\cdots=a_{n}^{e_{n}}=R^{m}\left(a_{1}, a_{2}, \ldots, a_{n}\right)=1\right\rangle
$$

with $n \geq 2, m \geq 2, e_{i}=0$ or $e_{i} \geq 2$ for $i=1, \ldots, n$ and $R\left(a_{1}, a_{2}, \ldots, a_{n}\right)$ a cyclically reduced word in the free product on $a_{1}, a_{2}, \ldots, a_{n}$ which involves all $a_{1}, \ldots, a_{n}$. Then

$$
\left\langle a_{1}, a_{2}, \ldots, a_{n-1}\right\rangle=\left\langle a_{1} ; a_{1}^{e_{1}}\right\rangle *\left\langle a_{2} ; a_{2}^{e_{2}}\right\rangle * \cdots *\left\langle a_{n} ; a_{n}^{e_{n}}\right\rangle,
$$

i.e. $\left\langle a_{1}, \ldots, a_{n-1}\right\rangle$ is the free product of cyclics of the obvious orders.

PROOF. The proof follows the technique of [1]. Without loss of generality we can assume $e_{i} \geq 2$ for $i=1, \ldots, n$.

Received by the editors November 25, 1985 and, in revised form, November 1, 1986.

1980 Mathematics Subject Classification (1985 Revision). Primary 20F05, 20E06; Secondary $20 \mathrm{E} 07$.

Key words and phrases. Freiheitssatz, one-relator product, generalized triangle group. 
First we choose projective matrices $A_{1}, A_{2}, \ldots, A_{n-1}$ in $\mathrm{PSL}_{2}(\mathrm{C})$ such that the subgroup generated by these is the appropriate free product of cyclics. That is

$$
\left\langle A_{1}, A_{2}, \ldots, A_{n-1}\right\rangle=\left\langle A_{1} ; A_{1}^{e_{1}}\right\rangle *\left\langle A_{2} ; A_{2}^{e_{2}}\right\rangle * \cdots *\left\langle A_{n-1} ; A_{n-1}^{e_{n-1}}\right\rangle
$$

and so $\left\langle A_{1}, A_{2}, \ldots, A_{n-1}\right\rangle$ faithfully represents the free product of cyclics $\left\langle a_{1}, \ldots\right.$, $\left.a_{n-1} ; a_{1}^{e_{1}}=\cdots=a_{n-1}^{e_{n-1}}=1\right\rangle$.

We will attempt to determine a projective matrix $A_{n} \in \mathrm{PSL}_{2}(\mathbf{C})$ of appropriate order $e_{n}$ so that the subgroup generated by $A_{1}, A_{2}, \ldots, A_{n}$ provides a representation of $G$ in $\mathrm{PSL}_{2}(\mathbf{C})$. Since the image of $a_{1}, a_{2}, \ldots, a_{n-1}$ will then be a free product of cyclics (each of maximal possible order), $\left\langle a_{1}, a_{2}, \ldots, a_{n-1}\right\rangle$ will also be the appropriate free product of cyclics.

Choose

$$
A_{n}=\left(\begin{array}{cc}
w & w t-w^{2}-1 \\
1 & t-w
\end{array}\right)
$$

where $t=2 \cos \left(\pi / e_{n}\right)$ and $w$ is to be determined. Since $\operatorname{tr}\left(A_{n}\right)=2 \cos \left(\pi / e_{n}\right)$ we have $A_{n}^{e_{n}}=1$ in $\mathrm{PSL}_{2}(\mathbf{C})$.

Consider the relator word $R\left(a_{1}, a_{2}, \ldots, a_{n}\right)$. Substituting $A_{1}, A_{2}, \ldots, A_{n}$ in $R$ we obtain the matrix

$$
R\left(A_{1}, A_{2}, \ldots, A_{n}\right)=\left(\begin{array}{ll}
f_{1} & f_{2} \\
f_{3} & f_{4}
\end{array}\right)
$$

where $f_{1}, f_{2}, f_{3}, f_{4}$ are polynomials in the coefficients of $A_{1}, A_{2}, \ldots, A_{n}$. Considering $w$ as the only unknown, $f_{1}, f_{2}, f_{3}, f_{4}$ are then polynomials in $w$.

If $\operatorname{tr}\left(R\left(A_{1}, A_{2}, \ldots, A_{n}\right)\right)=f_{1}+f_{4}$ is not a constant polynomial in $w$ then the polynomial equation

$$
f_{1}(w)+f_{4}(w)=2 \cos (\pi / m)
$$

can be solved for $w$.

For this choice of $w$ in $A_{n}$ we would have $R^{m}\left(A_{1}, A_{2}, \ldots, A_{m}\right)=1$ because of the trace. Therefore the subgroup generated by $A_{1}, A_{2}, \ldots, A_{n}$ provides a representation of $G$ in $\mathrm{PSL}_{2}(\mathrm{C})$ with $A_{1}, A_{2}, \ldots, A_{n-1}$ generating the appropriate free product of cyclics.

What is left in order to complete the proof is to show that there is a choice of $A_{1}, A_{2}, \ldots, A_{n-1}$ such that $\operatorname{tr}\left(R\left(A_{1}, A_{2}, \ldots, A_{n}\right)\right)$ is nonconstant in $w$.

Since $R\left(a_{1}, a_{2}, \ldots, a_{n}\right)$ is cyclically reduced we can write the relator $R$ without loss of generality as

$$
R\left(A_{1}, A_{2}, \ldots, A_{n}\right)=B_{1} A_{n}^{t_{1}} B_{2} \cdots B_{k} A_{n}^{t_{k}}
$$

with $B_{i}$ nontrivial words in $A_{1}, A_{2}, \ldots, A_{n-1}$ (hence nonidentity matrices) and $1 \leq t<e_{n}$.

Suppose $B_{1}=\left(\begin{array}{ll}a & b \\ c & d\end{array}\right) \neq \pm\left(\begin{array}{ll}1 & 0 \\ 0 & 1\end{array}\right)$.

Then $\left(\begin{array}{ll}1 & 0 \\ y & 1\end{array}\right) B_{1}\left(\begin{array}{cc}1 & 0 \\ -y & 1\end{array}\right)$ has lower left entry $c+y a-y d-y^{2} b$ which is nonzero for all but finitely many choices of $y$. Similarly for $B_{2}, \ldots, B_{k}$. Then by conjugating all the $B_{1}, B_{2}, \ldots, B_{k}$ by a suitable $\left(\begin{array}{ll}1 & 0 \\ y & 1\end{array}\right)$ if necessary we can assume that the lower left entries of $B_{1}, B_{2}, \ldots, B_{k}$ are nonzero. By considering the diagonalization of $A_{n}$ we see that $A_{n}^{t_{i}}$ has the form

$$
\left(\begin{array}{ll}
g_{1} & g_{2} \\
g_{3} & g_{4}
\end{array}\right)
$$


where $g_{1}, g_{2}, g_{3}, g_{4}$ are polynomials in $w$ of respective degrees $1,2,0$ and 1 . It follows that each factor $B_{i} A_{n}^{t_{i}}$ in the above expression for the relator $R$ must have the form

$$
\left(\begin{array}{ll}
h_{1} & h_{2} \\
h_{3} & h_{4}
\end{array}\right)
$$

where $h_{1}, h_{2}, h_{3}, h_{4}$ are also polynomials in $w$. Since each $B_{i}$ has nonzero lower left entry we must have the degree of $h_{4}$ is exactly 2 while the degrees of $h_{1}, h_{2}, h_{3}$ are $\leq 1, \leq 2, \leq 1$ respectively. Using an induction on $k \geq 1$ we can conclude that in $R$, $f_{1}$ and $f_{3}$ are of degree $<2 k, f_{2}$ is of degree $\leq 2 k$ and $f_{4}$ is of degree exactly $2 k$. This guarantees that $\operatorname{tr}\left(R\left(A_{1}, \ldots, A_{n}\right)\right)=f_{1}+f_{4}$ is nonconstant in $w$.

For the proper power case as in the above theorem the Freiheitssatz was known to hold if the order of the relator is 4 or greater-that is $R$ is cyclically reduced and $m \geq 4$. It was conjectured by Howie [3] that this could be weakened to $m \geq 2$. Thus Theorem 1 answers Howie's conjecture for the class of one-relator products of cyclics.

The proof of Theorem 1 depended on the fact that free products of cyclics can be faithfully represented in $\mathrm{PSL}_{2}(\mathrm{C})$. A straightforward extension then yields the following stronger result. Our main Theorem 1 can actually be considered as a special case of Theorem 2 .

THEOREM 2. Let $G=(A * B) / N\left(R^{m}\right)$ where $A$ and $B$ are groups admitting faithful representations in $\mathrm{PSL}_{2}(\mathbf{C})$, and $R$ is a cyclically reduced word in the free product of $A$ and $B$ of length $\geq 2$ and $m \geq 2$. Then $G$ admits a representation $\rho: G \rightarrow \mathrm{PSL}_{2}(\mathbf{C})$ such that $B \rightarrow G \stackrel{\rho}{\rightarrow} \mathrm{PSL}_{2}(\mathbf{C})$ and $A \rightarrow G \stackrel{\rho}{\rightarrow} \mathrm{PSL}_{2}(\mathbf{C})$ are faithful and $\rho(R)$ has order $m$. In particular $A \rightarrow G$ and $B \rightarrow G$ are injective-that is the Freiheitssatz holds.

PROOF. Write the relator as

$$
R=a_{1} b_{1} \cdots a_{k} b_{k} \quad \text { with } a_{i} \in A, b_{i} \in B .
$$

Since $R$ is cyclically reduced of length $\geq 2$ we can assume that $a_{i} \neq 1$ and $b_{i} \neq 1$.

Choose faithful representations $\rho_{A}: A \rightarrow \mathrm{PSL}_{2}(\mathbf{C})$ and $\rho_{B}: B \rightarrow \mathrm{PSL}_{2}(\mathbf{C})$ such that (after suitable conjugation if necessary)

$$
\begin{aligned}
& \rho_{A}\left(a_{i}\right)=\left(\begin{array}{cc}
* & x_{i} \\
* & *
\end{array}\right) \quad \text { with } x_{i} \neq 0 \\
& \rho_{B}\left(b_{i}\right)=\left(\begin{array}{cc}
* & y_{i} \\
* & *
\end{array}\right) \quad \text { with } y_{i} \neq 0
\end{aligned}
$$

for all $i=1, \ldots, k$.

For $w \in \mathbf{C}$ let $\rho_{A}^{w}$ denote the representation $\rho_{A}$ conjugated by $\left(\begin{array}{cc}1 & 0 \\ w & 1\end{array}\right)$. That is,

$$
\rho_{A}^{w}(a)=\left(\begin{array}{cc}
1 & 0 \\
w & 1
\end{array}\right) \rho_{A}(a)\left(\begin{array}{cc}
1 & 0 \\
-w & 1
\end{array}\right)
$$

Considering $w$ as a variable define

$$
T(w)=\operatorname{tr}\left(\rho_{A}^{w}\left(a_{1}\right) \rho_{B}\left(b_{1}\right) \cdots \rho_{A}^{w}\left(a_{k}\right) \rho_{B}\left(b_{k}\right)\right) .
$$

Then $T(w)$ is a polynomial in $w$. As in the proof of Theorem 1 the coefficient of $w^{2 k}$ is nonzero $\left(= \pm x_{1} y_{1} \cdots x_{k} y_{k}\right.$ ) because of the choices of $\rho_{A}$ and $\rho_{B}$. 
Then again as in the proof of Theorem 1 there exists a $w_{0}$ with

$$
T\left(w_{0}\right)=2 \cos (\pi / m) \text {. }
$$

Now define the representation $\rho: G \rightarrow \mathrm{PSL}_{2}(\mathbf{C})$ by $\left.\rho\right|_{A}=\rho_{A}^{w_{0}}$ and $\left.\rho\right|_{B}=\rho_{B}$. Then $\rho(R)$ has trace $T\left(w_{0}\right)=2 \cos (\pi / m)$ and so $\rho(R)$ has the correct order $m$. Further $\left.\rho\right|_{A}=\rho_{A}^{w_{0}}$ is faithful on $A$ and similarly $\left.\rho\right|_{B}$ is faithful on $B$. Thus $\rho$ is the desired representation.

Theorem 2 allows us to greatly extend the class of one-relator products for which the Freiheitssatz holds. If $G$ is a group of the form $(A * B) / N\left(R^{m}\right)$ with $R$ and $m$ as in the theorem then $A \rightarrow G$ and $B \rightarrow G$ are injective (the Freiheitssatz holds) whenever $A$ and $B$ are Kleinian groups, Fuchsian groups, Surface groups, free abelian groups of rank 2, free metabelian groups of rank 2 or fixed combinations of any of these. This is a consequence of the fact that each of these types of groups can be faithfully represented in $\mathrm{PSL}_{2}(\mathbf{C})$.

Using essentially the same technique as the proof of Theorem 1, Baumsiag, Morgan and Shalen investigated the generalized triangle groups. These are groups of the form $\left\langle a, b ; a^{m}=b^{p}=S^{n}(a, b)=1\right\rangle$ with $S(a, b)$ a word in $a$ and $b$. In the context of Theorem 1 these can be described as one-relator products of two cyclic groups. In particular they obtained the following two results.

Theorem A (BAumslag, Morgan And Shalen [1]). Let $G=\left\langle a, b ; a^{m}=\right.$ $\left.b^{p}=S^{n}(a, b)=1\right\rangle$ with $m, n, p \geq 2$. Then $G \neq\{1\}$.

TheOREM B (BAUMSlaG, MORGAN AND Shalen [1]). Let $G=\left\langle a, b ; a^{m}=\right.$ $\left.b^{p}=S^{n}(a, b)=1\right\rangle$ and let $W(G)=1 / m+1 / p+1 / n$. Then

(1) If $W(G) \leq 1$ then $G$ is infinite,

(2) If $W(G)<1$ then $G$ contains a free subgroup of rank 2 .

Theorem B mirrors the situation for the ordinary triangle groups - that is where $S(a, b)=a b$.

Part (2) of Theorem B raises the question of the nature of the nonabelian free subgroups of the generalized triangle groups. A result of Ree and Mendelsohn [6] shows that if $a$ and $b$ both have infinite order $(m=p=0)$ above and $S$ is a proper power involving both $a$ and $b$ then for sufficiently large $t$ the elements $a$ and $b^{t}$ generate a free subgroup of rank 2. Our methods allow us to extend this. First if one generator has infinite order:

COROLlaRY 1. Let $G=\left\langle a, b ; a^{m}=S^{n}(a, b)=1\right\rangle$ with $m \geq 2, n \geq 3$ and $S(a, b)$ a cyclically reduced word in the free product on $a$ and $b$ which involves both $a$ and $b$. Then there exists a generating pair $\{u, v\}$ of $G$ and a sufficiently large integer $t$ such that $\left\langle u^{t}, v^{t}\right\rangle$ is a free group of rank 2 .

From Theorem 1 we can find a representation of $G$ in $\mathrm{PSL}_{2}(\mathbf{C})$ where the image of $a$ has order $m \geq 2$, the image of $S$ has order $n$ and the image of $b$ has infinite order. The proof of Corollary 1 then involves a long technical argument on the possible types of images of $a$ and $b$ and their fixed points as mappings of the complex plane. This type of situation was handled in [7]. The details of the proof of Corollary 1 will appear elsewhere.

Using the same type of analysis we can handle the case where both generators have finite order and the relator is the commutator word $S(a, b)=[a, b]^{n}$. 
COROLlaRY 2. Let $G=\left\langle a, b ; a^{m}=b^{p}=[a, b]^{n}=1\right\rangle$ with $m=0$ or $m \geq 2$, $p=0$ or $p \geq 2, m \leq p$ and $n=0$ or $n \geq 2$. Suppose one of the following holds.

(i) $n=0$ and $p \geq 3$,

(ii) $m=0$,

(iii) $p=0$,

(iv) $m=3$ and $n \geq 3$ or $m=3$ and $p \geq 4$,

(v) $m=2, p \geq 3$ and $n \geq 4$ or $m=2, p \geq 4$ and $n \geq 3$,

(vi) $m=2$ and $p \geq 5$.

Then there exists a generating pair $\{u, v\}$ of $G$ and a sufficiently large integer $t$ such that $\left\langle u^{t}, v^{t}\right\rangle$ is a free group of rank 2 .

The proof of Corollary 2 depends, as in the last corollary, on the nature of the possible images of the generators of $G$ in $\mathrm{PSL}_{2}(\mathbf{C})$. The details will appear with those of Corollary 1.

Finally as a consequence of Theorem 1 we can give a relatively simple proof of the following theorem of Fischer, Karrass and Solitar [2].

COROLlaRY 3 (FISCHER, KARRASS, SOlITAR). Let $G=\left\langle a_{1}, a_{2}, \ldots, a_{n}\right.$; $\left.R^{m}\left(a_{1}, \ldots, a_{n}\right)\right\rangle$ with $m \geq 2$-that is $G$ is a one-relator group with torsion. Then $G$ contains a normal torsion-free subgroup of finite index.

PROOF. Without loss of generality we can assume that $R$ is cyclically reduced and involves all $a_{1}, \ldots, a_{n}$. Let $\rho: G \rightarrow \mathrm{PSL}_{2}(\mathbf{C})$ be the representation constructed as in Theorem 1 so that $\left.\rho\right|_{\left\langle a_{1}, \ldots, a_{n-1}\right\rangle}$ and $\left.\rho\right|_{\left\langle a_{n}\right\rangle}$ are faithful and $\rho\left(R\left(a_{1}, \ldots, a_{n}\right)\right)$ has order $m$.

Since $G$ is finitely generated, $\rho(G)$ is a finitely generated subgroup of $\mathrm{PSL}_{2}(\mathbf{C})$. From a theorem of Selberg [8], $\rho(G)$ contains a normal torsion-free subgroup of finite index. Call this subgroup $H . H$ then lifts to a normal subgroup $\bar{H}$ of finite index in $G$. We claim $\bar{H}$ is also torsion-free. If $g \in \bar{H}$ and $g$ has finite order then $g \in \operatorname{Ker} \rho$ since $H$ is torsion-free. But the elements of $G$ of finite order are all conjugates of powers of the relator $R[5]$. Since $\rho(R)$ has order $m$ it follows that Ker $\rho$ is torsion-free. Therefore $g=1$ and $\bar{H}$ is torsion-free.

Using a recent result of J. Howie [4] essentially the same proof gives the above result for one-relator quotients of locally indicable groups which admit faithful representations in $\mathrm{PSL}_{2}(\mathbf{C})$. Recall that a group $G$ is locally indicable if every finitely generated nontrivial subgroup of $G$ has an infinite cyclic homomorphic image. In particular,

COROLlARY 4. Let $G=(A * B) / N\left(R^{m}\right)$ where $A$ and $B$ are finitely generated locally indicable groups which admit faithful representations in $\mathrm{PSL}_{2}(\mathbf{C})$. Suppose $R$ is cyclically reduced in the free product $A * B$ of length $\geq 2$ and not a proper power in $A * B$. Then if $m \geq 2, G$ contains a torsion-free normal subgroup of finite index.

The proof depends as in Corollary 3 on the fact that elements of finite order are conjugates of powers of $R$. This was shown for groups satisfying the hypotheses of the corollary in [4]. We note also that Kleinian groups of the second kind are locally indicable and therefore the corollary above holds for one-relator products of finitely generated Kleinian groups of the second kind. We thank the referee for pointing this out to us. 


\section{REFERENCES}

1. G. Baumslag, J. Morgan and P. Shalen, On generalized triangle groups, presented at Groups St. Andrews, 1985 (to appear).

2. J. Fischer, A. Karrass and D. Solitar, On one-relator groups having elements of finite order, Proc. Amer. Math. Soc. 33 (1972), 297-301.

3. J. Howie, One-relator products of groups, Proceedings of Groups St. Andrews, St. Andrews, Scotland, 1985 (to appear).

4. _ Cohomology of one-relator products of locally indicable groups, J. London Math. Soc. (2) 30 (1984), 419-430.

5. A. Karrass, W. Magnus and D. Solitar, Elements of finite order in groups with a single defining relation, Comm. Pure Appl. Math. 13 (1968), 57-66.

6. R. Ree and N. S. Mendelsohn, Free subgroups of groups with a single defining relation, Arch. Math. 19 (1968), 577-580.

7. G. Rosenberger, On discrete free subgroups of linear groups, J. London Math. Soc. (2) 17 (1978), 79-85.

8. A. Selberg, On discontinuous groups in higher dimensional symmetric spaces, Internat. Colloq. Function Theory, Tata Institute, Bombay, 1960, pp. 147-164.

DePARTMENT OF MATHEMATICS, FAIRFIELD UNIVERSity, FAIRFIELD, CONNECTICUT 06430 (Current address of Benjamin Fine)

Department of Mathematics, University of Glasgow, Glasgow G12 80W, SCOTLAND

Abteiling Mathematik, Universitat Dortmund, Postfach 500, D-4600 DortMUND 50, FEDERAL REPUBLIC OF GERMANY (Current address of Gerhard Rosenberger)

Current address (James Howie): Department of Mathematics, Herriott Watt College, Glasgow, Scotland G12 80W 